\title{
A comparison of acyclovir and idoxuridine as treatment for ulcerative herpetic keratitis
}

\author{
DOUGLAS J. COSTER, K. R. WILHELMUS, R. MICHAUD, \\ AND BARRIE R. JONES \\ From the Department of Clinical Ophthalmology, Institute of Ophthalmology, \\ Moorfields Eye Hospital, City Road, London
}

SUMMARY Sixty patients were treated with either acyclovir $3 \%$ ointment or idoxuridine $1 \%$ ointment 5 times a day in a stratified randomised double-blind clinical trial. The 2 antiviral agents were equally effective.

Acyclovir (ACV, previously called Wellcome $248 \mathrm{U}$ and acycloguanosine) is a compound active in herpes simplex virus (HSV) infections. It has considerable clinical potential because it is extremely specific. Only cells infected with virus, and therefore with virus-coded thymidine kinase, phosphorylate the drug to a form capable of inhibiting DNApolymerase. Furthermore, it does not appear to be metabolised to inactive substances by human cells. ${ }^{1}$ The inhibition of viral coded DNA-polymerase is also highly specific. The potential value of the drug is related to its high specificity and low toxicity. Acyclovir has been demonstrated to possess potent antiviral activity in vitro and in animal models of herpetic keratitis. ${ }^{2}$

Clinical studies have confirmed the activity of the drug in human herpetic disease. The first of these studies involved debriding epithelial lesions produced by HSV and comparing the ability of acyclovir to prevent lesion formation. ${ }^{3}$ When dendritic ulcers are treated with debridement alone, healing is rapid and patients become free of pain within $24-48$ hours. However, $50 \%$ of patients so treated develop small foci of viral activity in the epithelium within a week of treatment that requires further debridement or antiviral chemotherapy. ${ }^{4}$ Placebo-controlled trials designed to test an antiviral agent's ability to suppress the formation of these microscopic lesions are capable of demonstrating a potentially useful biological activity while subjecting a very small number of patients to a small amount of drug.

Correspondence to Professor D. J. Coster, Department of Clinical Ophthalmology, Moorfields Eye Hospital, City Road, London ECIV 2PD.
The biological activity of acyclovir having been confirmed in human viral disease, the next step was to compare the drug used in a more conventional manner to established antiviral chemotherapy. Here we report the results of a trial comparing the effects of acyclovir and idoxuridine (IDU) ointments on the rate of healing of herpetic corneal ulcers.

\section{Patients and methods}

Sixty consenting patients presenting with ulcerative herpetic keratitis were admitted to the trial. Patients were allocated to strata on the basis of clinical features likely to be significant in prognosis. The stratification factors were the type of ulcer, that is, geographic or dendritic, the size of the ulcer, whether the patient had received topical corticosteroids within the 3 weeks prior to presentation, and the degree of inflammatory reaction in the cornea and anterior chamber. Within these strata patients were randomly allocated to 1 of 2 treatment groups. One group received IDU $1 \%$ ointment 5 times a day until the epithelial defect had healed and then 3 times a day for 3 more days. The other group received acyclovir 3\% ointment 5 times a day until the epithelial defect healed and then 3 times a day for a further 3 days. All patients received a drop of atropine $1 \%$ each day until healed. Neither the patient nor the ophthalmologist knew which treatment was being used, so that the trial was stratified, randomised, and double-blind.

Having begun treatment, patients were examined at the slit-lamp on alternate days. The point of healing was judged to have occurred when no epithelial defect was demonstrable with rose Bengal and fluorescein staining. 


\section{Results}

Sixty patients were admitted to the trial. Fifty-four had dendritic and 6 had geographic (amoeboid) ulcers. Thirty received IDU and 30 received acyclovir. The distribution of patients is set out in Table 1. One patient treated with acyclovir failed to present regularly for follow-up, though he responded favourably in that his geographic ulcer had healed when he returned 10 days after beginning therapy. $\mathrm{He}$ is not included in the analysis.

There were no treatment failures, nor were there any untoward reactions that necessitated withdrawal of therapy. One patient developed an allergic reaction that subsided on withdrawing the atropine drops.

The results were evaluated on the basis of the number of days it took the ulcers to heal in each group. The distribution of healing times for patients in both treatment groups is set out in Fig. 1. A cumulative frequency curve indicating the pattern of healing in each group is displayed in Fig. 2. Clearly the patterns are very similar, and a logrank analysis indicates that the difference between the

Table 1 Distribution of patients with dendritic or geographic ulcers to treatment group to receive idoxuridine (IDU) or acyclovir $(A C V)$

\begin{tabular}{lcc}
\hline & $I D U$ & $A C V$ \\
\hline Geographic & 4 & $2 *$ \\
Small dendritic & $14^{*}$ & $14^{*}$ \\
Large dendritic & 12 & 14 \\
Total & 30 & 30
\end{tabular}

- Patients with marked inflammatory reaction in the corneal stroma and anterior chamber.

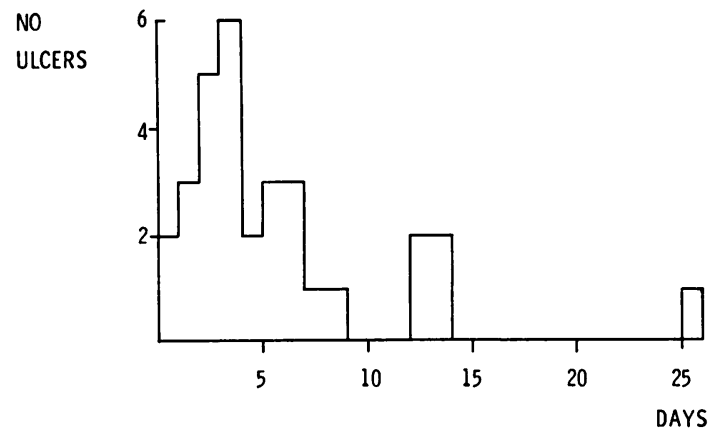

Fig. 1 Histogram of healing times of herpetic ulcers treated with acyclovir ( $A C V)$ and 5-iodo-2' deoxyuridine $(I D U)$. curves is likely to have occurred by change $(\mathrm{P}>0.05)$. When the geographic ulcers are excluded from the analysis the difference between the groups is even less (Fig. 3).

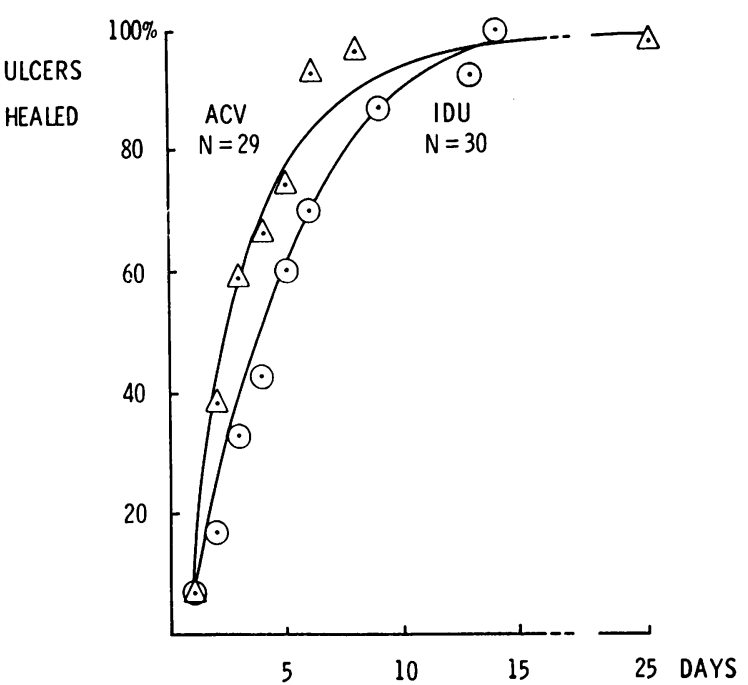

Fig. 2 Cumulative frequency graph of the time taken to heal of 59 herpetic corneal ulcers (54 dendritic, 5 geographic) treated with acyclovir $(A C V)$ or 5-iodo-2' deoxyuridine (IDU).

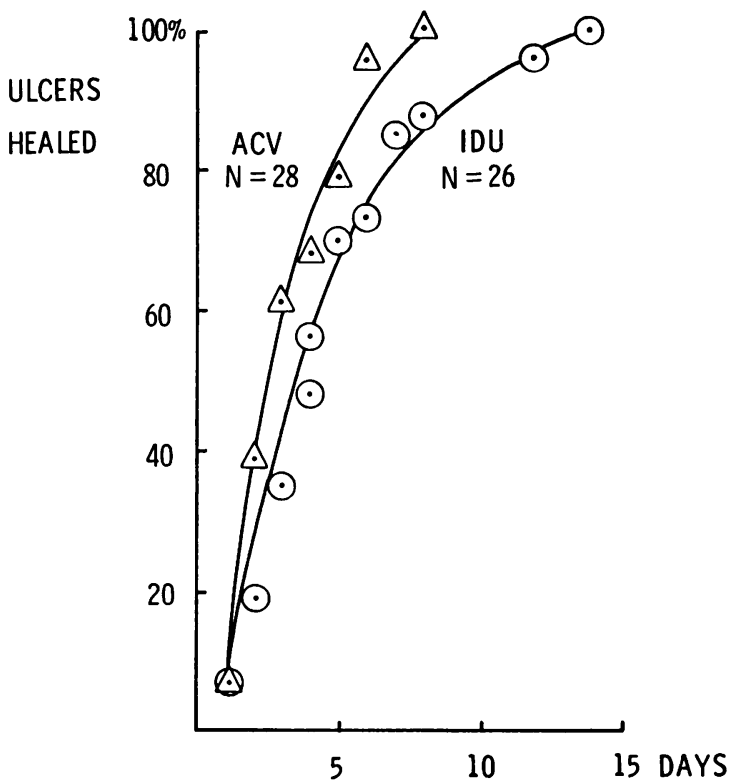

Fig. 3 Cumulative frequency graph of the time taken to heal of 54 dendritic ulcers treated with acyclovir ( $A C V)$ or 5-iodo-2' deoxyuridine (IDU). 


\section{Discussion}

The results of this trial establish that acyclovir is an effective antiviral agent for treating ulcerative herpetic keratitis, since it is at least as active as IDU.

It is to be expected that such a trial will yield a null result. The majority of ulcers are dendritic rather than geographic. IDU is so effective for dendritic ulcers, with $100 \%$ of patients being healed within 14 days, that there is little chance of another antiviral agent bringing about a significant improvement on what is achieved with IDU. This is not to say that a null result is insignificant, but it demands that trials be well designed, carefully conducted, and the data be analysed in an appropriate manner.

A further limitation of trials evaluating antiviral agents as treatment for dendritic ulcers is that they do not provide any information on chronic toxicity, as the majority of patients complete the treatment programme in less than 2 weeks. This is a major limitation, since it is the toxicity of antivirals that limits their clinical usefulness.

Since it is established that acyclovir is as effective as IDU in the treatment of ulcerative herpetic keratitis, the next step is to evaluate the drug in situations in which currently employed therapeutic measures are less than universally satisfactory. Geographic ulcers are generally more difficult to treat than dendritic ulcers. They have been shown to represent a different level of challenge and are capable of demonstrating a difference between antiviral agents that cannot be appreciated when only dendritic ulcers are studied. ${ }^{5}$ Herpetic corneal ulcers which have failed to heal with IDU or debridement are another difficult problem with which to challenge a prospective new topical antiviral agent. Stromal keratitis is also a form of herpetic eye disease often resistant to current therapy. An important challenge is to conduct trials directed at evaluating this new antiviral agent in these sight-threatening conditions. Because of its remarkably low toxicity and high specificity, acyclovir is a drug with exciting therapeutic potential.

\section{References}

1 Schaeffer HJ, de Miranda P, Elion GB, Bauer JD, Collins P. 9-(Hydroxyethoxymethyl) guanine activity against viruses in the herpes group. Nature 1978; 272: 583-5.

2 Falcon MG, Jones BR. Acycloguanosine: antiviral activity in the rabbit cornea. Br J Ophthalmol 1979; 63: 422-4.

3 Coster DJ, Jones BR, Falcon MG. Role of debridement in the treatment of herpetic keratitis. Trans Ophthalmol Soc UK 1977; 97: 314-7.

4 Jones BR, Coster DJ, Fison PN, Thompson GM, Cobo LM, Falcon MG. Efficacy of acycloguanosine (Wellcome 248U) against herpes simplex corneal ulcers. Lancet 1979; i: $243-4$.

5 Coster DJ, Jones BR, McGill JI. Treatment of amoeboid herpetic ulcers with adenine arabinoside or trifluorothymidine. Br J Ophthalmol 1979; 63: 418-21. 